\title{
GENERALIZED NAVIER BOUNDARY CONDITION FOR THE MOVING CONTACT LINE*
}

\author{
TIEZHENG QIAN ${ }^{\dagger}$, XIAO-PING WANG ${ }^{\ddagger}$ AND PING SHENG $\sharp$
}

\begin{abstract}
From molecular dynamics simulations on immiscible flows, we find the relative slipping between the fluids and the solid wall everywhere to follow the generalized Navier boundary condition, in which the amount of slipping is proportional to the sum of tangential viscous stress and the uncompensated Young stress. The latter arises from the deviation of the fluid-fluid interface from its static configuration. We give a continuum formulation of the immiscible flow hydrodynamics, comprising the generalized Navier boundary condition, the Navier-Stokes equation, and the CahnHilliard interfacial free energy. Our hydrodynamic model yields near-complete slip of the contact line, with interfacial and velocity profiles matching quantitatively with those from the molecular dynamics simulations.
\end{abstract}

\section{Introduction}

Immiscible two-phase flow in the vicinity of the contact line (CL), where the fluid-fluid interface intersects the solid wall, is a classical problem that falls beyond the framework of conventional hydrodynamics $[1,2,3,4,5,6,7,8,9,10,11,12,13,14]$. In particular, molecular dynamics (MD) studies have shown relative slipping between the fluids and the wall, in violation of the no-slip boundary conditions $[6,7,8,9]$. While there have been numerous ad-hoc models $[1,10,12,13,14]$ to address this phenomenon, none has been able to give a quantitative account of the MD slip velocity profile in the molecular-scale vicinity of the CL. This failure casts doubts on the general applicability of the continuum hydrodynamics in the CL region. In particular, a (possible) breakdown in the hydrodynamic description for the molecular-scale CL region has been suggested $[8,9]$. Without a continuum hydrodynamic formulation, it becomes difficult or impossible to have realistic simulations of micro- or nanofluidics, or of immiscible flows in porous media where the relative wetting characteristics, the moving CL dissipation, and behavior over undulating surfaces may have macroscopic implications.

From MD simulations on immiscible two-phase flows, we report the finding that the generalized Navier boundary condition (GNBC) applies for all boundary regions, whereby the relative slipping is proportional to the sum of tangential viscous stress and the uncompensated Young stress. The latter arises from the deviation of the fluidfluid interface from its static configuration [12]. By combining GNBC with the CahnHilliard $(\mathrm{CH})$ hydrodynamic formulation of two-phase flow $[13,14]$, we obtained a consistent, continuum description of immiscible flow with predictions matching those from MD simulations. Our findings suggest the no-slip boundary condition to be an approximation to the GNBC, accurate for most macroscopic flows but failing in immiscible flows.

\section{Molecular dynamics simulations}

The MD simulations were performed for both the static and dynamic configurations in Couette and Poiseuille flows [15]. The two immiscible fluids were confined

\footnotetext{
* Received: October 6, 2002; accepted (in revised version): October 14, 2002.

$\dagger$ Department of Physics and Institute of Nano Science and Technology, The Hong Kong University of Science and Technology, Clear Water Bay, Kowloon, Hong Kong.

$\ddagger$ Department of Mathematics, The Hong Kong University of Science and Technology, Clear Water Bay, Kowloon, Hong Kong.

\# Department of Physics and Institute of Nano Science and Technology, The Hong Kong University of Science and Technology, Clear Water Bay, Kowloon, Hong Kong.
} 
between two parallel walls separated along the $z$ direction, with the fluid-solid boundaries defined by $z=0, H$. Interaction between the fluid molecules was modeled by a modified Lennard-Jones (LJ) potential $U_{f f}=4 \epsilon\left[(\sigma / r)^{12}-\delta_{f f}(\sigma / r)^{6}\right]$, where $r$ is the distance between the molecules, $\epsilon$ and $\sigma$ are the energy scale and range of interaction, respectively, and $\delta_{f f}=1$ for like molecules and $\delta_{f f}=-1$ for molecules of different species. Each of the two walls was constructed by two [001] planes of an fcc lattice, with each wall molecule attached to the lattice site by a harmonic spring. The mean square displacement of wall molecules was controlled to obey the Lindemann criterion. The wall-fluid interaction was also modeled by a LJ potential $U_{w f}$, with energy and range parameters $\epsilon_{w f}=1.16 \epsilon$ and $\sigma_{w f}=1.04 \sigma$, and a $\delta_{w f}$ for specifying the wetting property of the fluid. The mass of the wall molecule was set equal to that of the fluid molecule $m$, and the average number densities for the fluids and wall were set at $\rho=0.81 / \sigma^{3}$ and $\rho_{w}=1.86 / \sigma^{3}$, respectively. The temperature was controlled at $2.8 \epsilon / k_{B}$, where $k_{B}$ is Boltzmann's constant. Moving the top and bottom walls at a constant speed $V$ in the $\pm x$ directions, respectively, induced the Couette flow $[8,9]$. Applying a body force $m \mathbf{g}_{e x t}$ to each fluid molecule in the $x$ direction induced the Poiseuille flow $[6,7]$. Periodic boundary conditions were imposed on the $x$ and $y$ boundaries of the sample. Most of our MD simulations were carried out on samples consisting 6144 atoms for each fluid and 2880 atoms for each wall. The sample is $163.5 \sigma$ by $6.8 \sigma$ along the $x$ and $y$, respectively, and $H=13.6 \sigma$. Our MD results represent time averages over 20 to 40 million time steps.

Two different cases were considered in our simulations. The symmetric case refers to identical wall-fluid interactions for the two fluids (both $\delta_{w f}=1$ ), which leads to a flat static interface in the $y z$ plane with a $90^{\circ}$ contact angle. The asymmetric case refers to different wall-fluid interactions, with $\delta_{w f}=1$ for one and $\delta_{w f}=0.7$ for the other. The resulting static interface is a circular arc with a $64^{\circ}$ contact angle. We measured six quantities in the Couette-flow steady states of $V=0.25(\epsilon / m)^{1 / 2}$, $H=13.6 \sigma$ for the symmetric case: $v_{x}^{\text {slip }}$, the slip velocity relative to the moving wall; $G_{x}^{w}$, the tangential force per unit area exerted by the wall; the $\sigma_{x x}, \sigma_{n x}$ components of the fluid stress tensor ( $n$ denotes the outward surface normal), and $v_{x}, v_{z}$. We denote the region within $0.85 \sigma=z_{0}$ of the wall the boundary layer (BL). It must be thin enough to render sufficient precision for measuring $v_{x}^{\text {slip }}$ and meanwhile be thick enough to fully account for the tangential wall-fluid interaction force, due to the finite range of the LJ interaction. The wall force can be identified by separating the force on each fluid molecule into wall-fluid and fluid-fluid components. For $0<z \leq z_{0}$ the fluid molecules can detect the atomic structure of the wall. When coupled with kinetic collisions with the wall molecules, there arises a nonzero tangential wall force that varies along the $z$ direction and saturates at $z \simeq z_{0}$. $G_{x}^{w}$ (both static and dynamic) is the saturated total tangential wall force per unit wall area. It is not possible to do MD measurements strictly at the fluid-solid boundary, not only because of poor statistics, but also because of this intrinsic limitation. Spatial resolution along the $x$ and $z$ directions was achieved by evenly dividing the sampling region into bins, each $\Delta x=0.425 \sigma$ by $\Delta z=0.85 \sigma$ in size. $v_{x}^{\text {slip }}$ was obtained as the time average of fluid molecules' velocities inside the BL, measured with respect to the moving wall; $G_{x}^{w}$ was obtained from the time average of the total tangential wall force experienced by the fluid molecules in the $\mathrm{BL}$, divided by the bin area in the $x y$ plane; $\sigma_{x x(n x)}$ was obtained from the time averages of the momentum transfer plus the fluid-fluid interaction forces across the constant- $x(z)$ bin surfaces, and $v_{x(z)}$ was measured as the time-averaged velocity component(s) within each bin. For the contribution of intermolecular forces 
to the stress, we have directly measured the fluid-fluid interaction forces across bin surfaces instead of using the Irving-Kirkwood expression [16], whose validity was noted to be not justified at the fluid-fluid or the wall-fluid interface (see the paragraph following equation (5.15) in the above reference). As reference quantities, we also measured $G_{x}^{w 0}, \sigma_{x x}^{0}, \sigma_{n x}^{0}$ in the static $(V=0)$ configuration. In addition, the average molecular densities $\rho_{1}$ and $\rho_{2}$ for the two fluid species in each bin, as well as the shear viscosity $\eta=1.95 \sqrt{\epsilon m} / \sigma^{2}$ and the interfacial tension $\gamma=5.5 \epsilon / \sigma^{2}$ were also determined.

We have also measured the interface and velocity profiles for the asymmetric case in the Poiseuille geometry, with $m g_{e x t}=0.05 \epsilon / \sigma$ applied in the $x$ direction and $H=13.6 \sigma$.

We present evidence to show that everywhere on the boundaries, relative slipping is proportional to the net tangential force per unit area exerted on the three (fluid) sides of the BL fluid element, due to the hydrodynamic motion of the surrounding fluid(s) (the GNBC, see also equation (2.3) below):

$$
\tilde{G}_{x}^{f}=\beta v_{x}^{s l i p}
$$

where $\beta$ is the slip coefficient, over tilde denotes the quantity to be the difference between the dynamic and static values (e.g., $\left.\tilde{\sigma}_{x x(n x)}=\sigma_{x x(n x)}-\sigma_{x x(n x)}^{0}\right)$, and $\tilde{G}_{x}^{f}$ denotes

$$
\tilde{G}_{x}^{f}=\partial_{x} \int_{0}^{z_{0}} \tilde{\sigma}_{x x}(z) d z-\tilde{\sigma}_{n x}\left(z_{0}\right)
$$

obtained by integrating from 0 to $z_{0}$ the $x$-direction hydrodynamic force density, $\partial_{x} \tilde{\sigma}_{x x}+\partial_{z} \tilde{\sigma}_{z x}$, and noting $\tilde{\sigma}_{n x}(0)=0$ at the wall-fluid boundary (strictly it is $\tilde{\sigma}_{n x}\left(0^{-}\right)=0$ ). Here the $z$ coordinate is for the lower fluid-wall boundary (same below), with the understanding that the same physics holds at the upper boundary, and $\partial_{x, z, n}$ means taking partial derivative with respect to $x$, $z$, or surface normal $n$ $\left(\partial_{n}=-\partial_{z}\right.$ for the lower boundary). We have verified the steady state force balance $\tilde{G}_{x}^{w}+\tilde{G}_{x}^{f}=0$ on the two boundaries (inset to figure 2.1). Inertial effect was found to be less than $\sigma_{n x}$ by two orders of magnitude. In figure 2.1 we show the measured MD data for the symmetric case in the Couette geometry. The solid lines represent the values of $\tilde{G}_{x}^{f}$ calculated from $\beta v_{x}^{\text {slip }}$ by using $\beta=\beta_{1}=\beta_{2}=1.2 \sqrt{\epsilon m} / \sigma^{3}$. For the asymmetric case, independent measurement determines $\beta_{1}=1.2 \sqrt{\mathrm{\epsilon m}} / \sigma^{3}$, $\beta_{2}=0.532 \sqrt{\epsilon m} / \sigma^{3}$, and $\beta=\left(\beta_{1} \rho_{1}+\beta_{2} \rho_{2}\right) /\left(\rho_{1}+\rho_{2}\right)$ in the CL region, with $\tilde{G}_{x}^{f}$, $v_{x}^{\text {slip }}$, and $\rho_{1,2}$ obtained from MD simulations. Here $\beta=\left(\beta_{1} \rho_{1}+\beta_{2} \rho_{2}\right) /\left(\rho_{1}+\rho_{2}\right)$ is obtained by assuming the two fluids interacting independently with the wall, so that $\tilde{G}_{x}^{f}$ may be expressed as the weighted average of $\tilde{G}_{x}^{f 1}=\beta_{1} v_{x}^{\text {slip } 1}$ and $\tilde{G}_{x}^{f 2}=\beta_{2} v_{x}^{\text {slip2 }}$. The desired expression is obtained by noting $v_{x}^{\text {slip } 1} \simeq v_{x}^{\text {slip } 2}$ to within $10 \%$.

The fact that the wall force density is distributed inside a thin BL and vanishes beyond the BL necessitates the form of $\tilde{G}_{x}^{f}$ as defined by equation (2.2). However, it is intuitively obvious that the fluids would experience almost the identical physical effect(s) from a wall force density $\tilde{G}_{x}^{w} \delta(z)$, concentrated strictly at the fluid-solid boundary with the same total wall force per unit area. The replacement of a diffuse boundary by a sharp boundary can considerably simplify the form of the GNBC, because local force balance along $x$ then requires $\partial_{x} \tilde{\sigma}_{x x}+\partial_{z} \tilde{\sigma}_{z x}=0$ in the fluid. Integration of this relation from 0 to $z_{0}$ yields $\tilde{G}_{x}^{f}=-\tilde{\sigma}_{n x}\left(0^{+}\right)$(by comparing with equation (2.2)). Thus, the GNBC (2.1) becomes $-\tilde{\sigma}_{n x}(0)=\beta v_{x}^{\text {slip }}$ in the sharp boundary limit. 


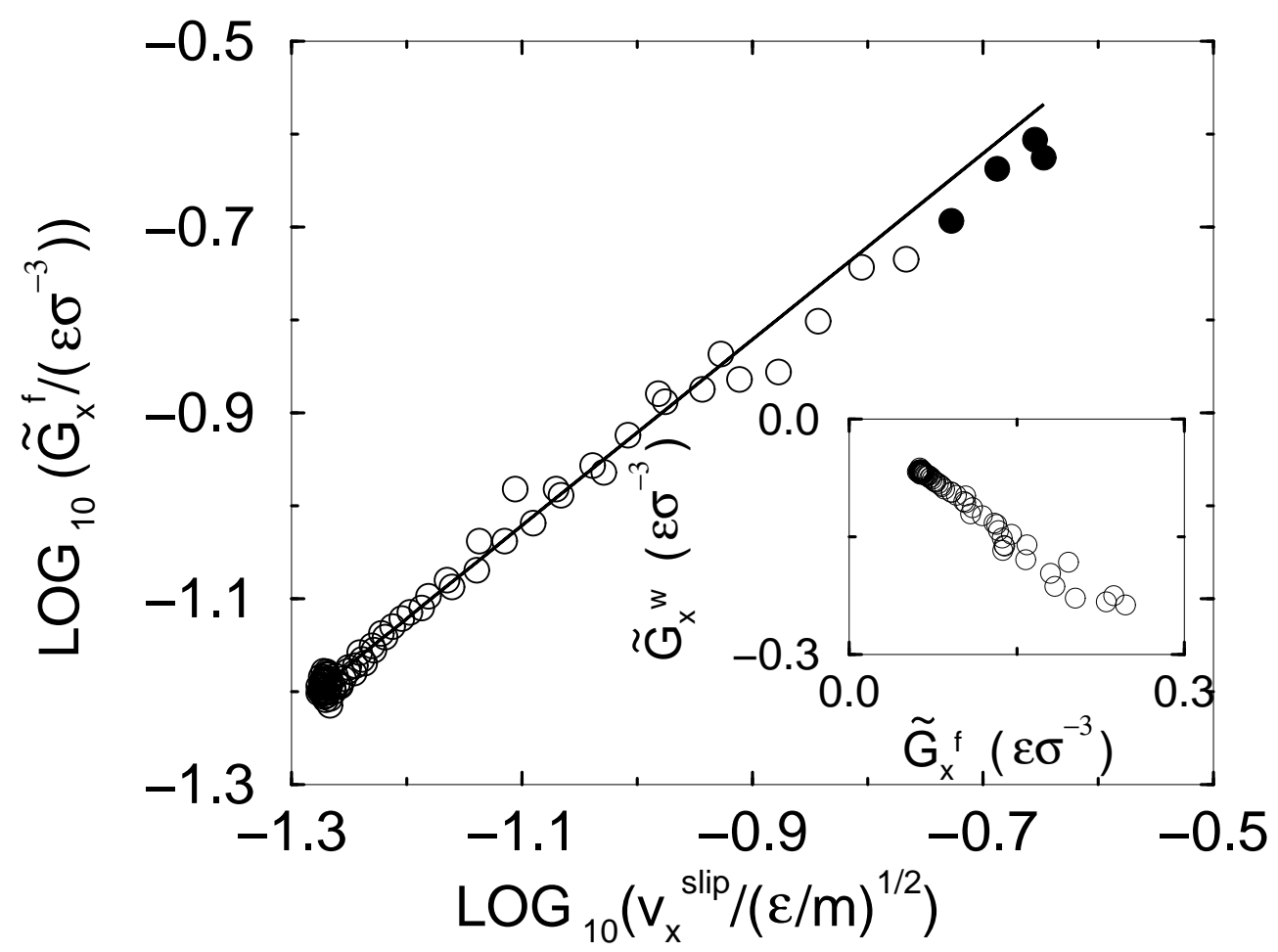

FIG. 2.1. $\tilde{G}_{x}^{f}$ plotted as a function of $v_{x}^{\text {slip }}$. Here we show the results obtained for the Couette flow in the symmetric case $\left(V=0.25(\epsilon / m)^{1 / 2}\right.$ and $\left.H=13.6 \sigma\right)$. Symbols are $M D$ data measured in the BL at different $x$ locations. The solid lines were calculated from equation (2.1) with values of $\beta_{1,2}$ (and the expression for $\beta$ ) given in the text. The statistical errors of the MD data are about the size of the symbols. Since in the present case the data for the lower and upper boundaries are related by symmetry, we show only the data for the lower boundary. Solid symbols indicate those data points inside the interfacial region, in the vicinity of the CL. The fact that the measured data lie on a straight line (with a slope of 1 in log-log plot) indicates the validity of the GNBC. Inset: $\tilde{G}_{x}^{w}$ plotted as a function of $\tilde{G}_{x}^{f}$, measured in the the lower $B L$ at different values of $x$. The data are seen to lie on a straight line with a slope of -1 , indicating $\tilde{G}_{x}^{w}+\tilde{G}_{x}^{f}=0$.

The tangential stress $\tilde{\sigma}_{n x}$ can be decomposed into a viscous component and a non-viscous component: $\tilde{\sigma}_{n x}(z)=\sigma_{n x}^{v}(z)+\tilde{\sigma}_{n x}^{Y}(z)$. In figure 2.2 , we show that away from the interfacial region the tangential viscous stress $\sigma_{n x}^{v}(z)=\eta\left(\partial_{n} v_{x}+\partial_{x} v_{n}\right)(z)$ is the only nonzero component, but in the interfacial region $\tilde{\sigma}_{n x}^{Y}=\sigma_{n x}-\sigma_{n x}^{v}-\sigma_{n x}^{0}=$ $\sigma_{n x}^{Y}-\sigma_{n x}^{0}$ is dominant. Therefore, away from the CL region the Navier Boundary Condition (NBC) is valid [17], but in the interfacial region the NBC clearly fails to describe the CL motion. We wish to clarify the origin of $\sigma_{n x}^{Y}$ and $\sigma_{n x}^{0}$ as the dynamic and static Young stresses, respectively, so that $\tilde{\sigma}_{n x}^{Y}=\sigma_{n x}^{Y}-\sigma_{n x}^{0}$ is the uncompensated Young stress. As shown in the inset to figure 2.2, the integrals (across the interface) of $\sigma_{n x}^{Y}$ ( $=\sigma_{n x}-\sigma_{n x}^{v}$, calculated by subtracting the viscous component $\eta\left(\partial_{n} v_{x}+\partial_{x} v_{n}\right)$ from the total tangential stress) and $\sigma_{n x}^{0}$ are equal to $\gamma \cos \theta_{d}$ and $\gamma \cos \theta_{s}$, respectively, at different values of $z$, i.e., $-\int_{\text {int }} \sigma_{n x}^{Y}(z) d x=\gamma \cos \theta_{d}(z)$ and $-\int_{\text {int }} \sigma_{n x}^{0}(z) d x=\gamma \cos \theta_{s}(z)$, where $\theta_{d}(z)$ and $\theta_{s}(z)$ are respectively the dynamic and static interfacial angles at $z$. These results clearly show the origin of the extra 
tangential stress in the interfacial region to be from interfacial (uncompensated) Young stress. Thus, the GNBC is given by

$$
\beta v_{x}^{s l i p}=-\tilde{\sigma}_{n x}(0)=-\left[\eta \partial_{n} v_{x}\right](0)-\tilde{\sigma}_{n x}^{Y}(0) .
$$

Here only one component of the viscous stress is nonzero, due to $v_{n}=0$ at the boundary; and $-\tilde{\sigma}_{n x}^{Y}(0)$ is denoted the uncompensated Young stress, satisfying $-\int_{\text {int }} \tilde{\sigma}_{n x}^{Y}(0)$ $d x=\gamma\left(\cos \theta_{d}^{0}-\cos \theta_{s}^{0}\right)$, with $\theta_{d(s)}^{0}$ being a microscopic dynamic (static) contact angle at the fluid-solid boundary. The fact that $\tilde{\sigma}_{n x}^{Y}(0) \simeq 0$ away from the CL shows that the GNBC implies NBC for single phase flows. In essence, our results show that in the vicinity of the CL, the tangential viscous stress $-\sigma_{n x}^{v}$ as postulated by the NBC cannot give rise to the near-complete CL slip without taking into account the tangential Young stress $-\sigma_{n x}^{Y}$ in combination with the gradient of (BL-integrated) normal stress $\sigma_{x x}$. For the static configuration, the normal stress gradient is balanced by the Young stress, leading to the Young equation. It is only for a moving CL that there is a component of the Young stress which is no longer balanced by the normal stress gradient, and this uncompensated Young stress is precisely the additional component captured by the GNBC but missed by the NBC.

\section{Continuum hydrodynamic formulation}

Based on the MD results, we formulate a (first approximation) hydrodynamic model based on the GNBC and the $\mathrm{CH}$ free energy functional [18]. The $\mathrm{CH}$ free energy is noted to be successful in the calculations of fluid-fluid interfacial phenomena:

$$
F=\int d \mathbf{r}\left[\frac{1}{2} K(\nabla \phi)^{2}+f(\phi)\right]
$$

where $\phi=\left(\rho_{2}-\rho_{1}\right) /\left(\rho_{2}+\rho_{1}\right), f(\phi)=-\frac{1}{2} r \phi^{2}+\frac{1}{4} u \phi^{4}$, and $K, r, u$ are parameters which can be directly obtained from MD simulations through the interface profile thickness $\xi=\sqrt{K / r}$, the interfacial tension $\gamma=2 \sqrt{2} r^{2} \xi / 3 u$, and the two homogeneous equilibrium phases given by the condition of $\partial f / \partial \phi=0$, yielding $\phi_{ \pm}= \pm \sqrt{r / u}$ $(= \pm 1$ in our case). From MD simulations, the interface $\xi \simeq 0.3 \sigma$ has a profile very accurately described by the $\tanh (x / \sqrt{2} \xi)$ form predicted by the $\mathrm{CH}$ free energy. The small value of $\xi$ implies negligible diffusion across the interface.

A displacement of the molecules from $\mathbf{r}$ to $\mathbf{r}^{\prime}=\mathbf{r}+\mathbf{u}(\mathbf{r})$ induces a local change of $\phi, \delta \phi=-\mathbf{u} \cdot \nabla \phi$, to the first order in $\mathbf{u}$. The associated change in $F$ is given by $\delta F=-\int d \mathbf{r}[\mathbf{g} \cdot \mathbf{u}]+\int d s\left[\sigma_{n i}^{Y} u_{i}\right]$, where $\mathbf{g}=\mu \nabla \phi$ is the capillary force density, with $\mu=\delta F / \delta \phi$ being the chemical potential, and $\sigma_{n i}^{Y}=-K \partial_{n} \phi \partial_{i} \phi$ is the tangential Young stress due to the spatial variation of $\phi$ at the fluid-solid boundary. Hence the two coupled equations of motion are the Navier-Stokes equation (with the addition of the capillary force density) and the convection-diffusion equation for $\phi(\mathbf{r})$ :

$$
\begin{gathered}
\rho_{m}\left[\frac{\partial \mathbf{v}}{\partial t}+(\mathbf{v} \cdot \nabla) \mathbf{v}\right]=-\nabla p+\nabla \cdot \boldsymbol{\sigma}^{v}+\mu \nabla \phi+\rho_{m} \mathbf{g}_{\text {ext }}, \\
\frac{\partial \phi}{\partial t}+\mathbf{v} \cdot \nabla \phi=M \nabla^{2} \mu,
\end{gathered}
$$

together with the incompressibility condition $\nabla \cdot \mathbf{v}=0$. Here $\rho_{m}$ is the fluid mass density, $p$ is the pressure, $\boldsymbol{\sigma}^{v}$ denotes the viscous part of the stress tensor, $\rho_{m} \mathbf{g}_{e x t}$ is 


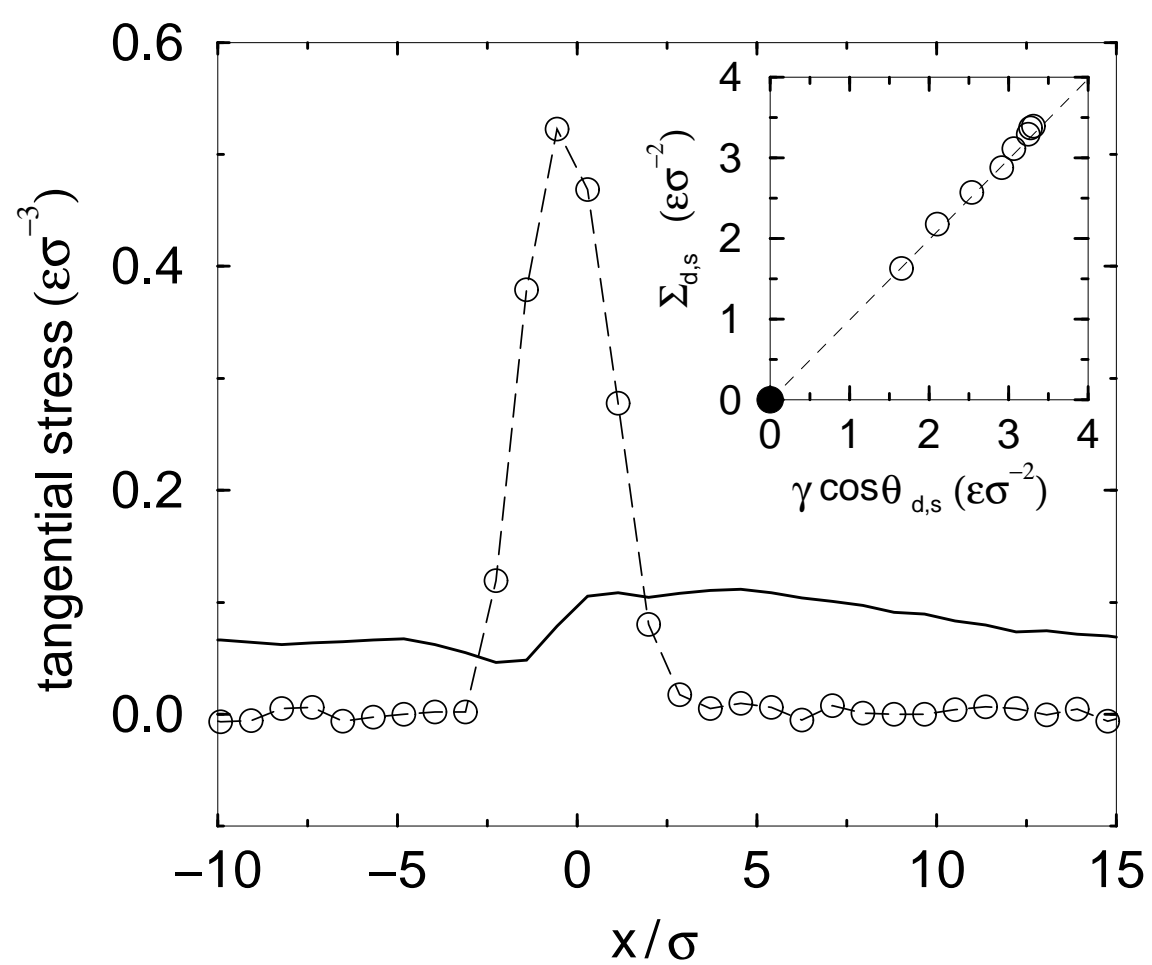

FIG. 2.2. Two components of the dynamic tangential stress at $z=z_{0}$, plotted as a function of $x$. Here we show the results obtained for the Couette flow in the symmetric case $\left(V=0.25(\epsilon / m)^{1 / 2}\right.$ and $H=13.6 \sigma)$.Similar results are also obtained for the asymmetric case. The dashed lines denote $\tilde{\sigma}_{z x}^{Y}$; solid lines represent the viscous component. In the CL region, the non-viscous component is one order of magnitude larger than the viscous component. The difference between the two components, however, diminishes towards the boundary, $z=0$, due to the large interfacial pressure drop (implying a large curvature) in the BL, thereby pulling $\theta_{d}$ closer to $\theta_{s}$. Inset: $\Sigma_{d, s}$ plotted as a function of $\gamma \cos \theta_{d, s}$ at different values of $z$. Here $\Sigma_{d}=-\int d x\left(\sigma_{n x}-\sigma_{n x}^{v}\right), \Sigma_{s}=-\int d x \sigma_{n x}^{0}$, and $\theta_{d, s}$ was measured from the time-averaged interfacial profiles. The hollow circles denote the dynamic case and the single solid circle at the origin denotes the static case. The data are seen to follow a straight (dashed) line with slope 1, indicating $\Sigma_{d, s}=\gamma \cos \theta_{d, s}$. This is also found to be true for the asymmetric case.

the external body force density (for Poiseuille flows), and $M$ is the phenomenological mobility coefficient.

Four boundary conditions are required. Two are given by the impermeability condition, i.e., the normal components of the fluid velocity and diffusive flux are zero $\left(v_{n}=0\right.$ and $\left.\partial_{n} \mu=0\right)$. The other two boundary conditions may be derived from our knowledge of CNBC plus the total free energy $F[\phi]+\int d s \gamma_{w f}(\phi)$, where $\gamma_{w f}(\phi)$ is the interfacial free energy at the fluid-solid boundary. We use $\gamma_{w f}(\phi)=\left(\Delta \gamma_{w f} / 2\right) \sin (\pi \phi / 2)$ to denote a smooth interpolation between $\pm \Delta \gamma_{w f} / 2$, with $\Delta \gamma_{w f}=-\gamma \cos \theta_{s}^{0}$ (Young's equation). Here it should be noted that the form of the smooth interpolation has very little effect on the final results. Hence, we have chosen a very simple interpolation function. The continuum form of the GNBC (2.3) 
is given by

$$
\beta v_{x}^{\text {slip }}=-\tilde{\sigma}_{n x}(0)=-\eta\left[\partial_{n} v_{x}\right](0)+\left[L(\phi) \partial_{x} \phi\right](0),
$$

where $\left[L(\phi) \partial_{x} \phi\right](0)$, with $L(\phi)=K \partial_{n} \phi+\partial \gamma_{w f}(\phi) / \partial \phi$, is the differential expression for $-\tilde{\sigma}_{n x}^{Y}(0)=-\sigma_{n x}^{Y}(0)+\sigma_{n x}^{0}(0)$ in equation (2.3) [14]. Here $K \partial_{n} \phi \partial_{x} \phi$ is $-\sigma_{n x}^{Y}(0)$ and $\left[\partial \gamma_{w f}(\phi) / \partial \phi\right] \partial_{x} \phi=\partial_{x} \gamma_{w f}(\phi)$ is $\sigma_{n x}^{0}(0)$ (from static force balance $\partial_{x} \gamma_{w f}(\phi)-$ $\left.\sigma_{n x}^{0}(0)=0\right)$, with $\int_{\text {int }} d x\left[K \partial_{n} \phi \partial_{x} \phi\right](0)=\gamma \cos \theta_{d}^{0}$ and $\int_{\text {int }} d x \partial_{x} \gamma_{w f}=-\gamma \cos \theta_{s}^{0}$, thus $\int_{\text {int }} d x\left[L(\phi) \partial_{x} \phi\right](0)$ equals to $\gamma\left(\cos \theta_{d}^{0}-\cos \theta_{s}^{0}\right)$, with $\left[L(\phi) \partial_{x} \phi\right](0)$ being the uncompensated Young stress.

Another boundary condition may be inferred from the fact that $L(\phi)=0$ is the Euler-Lagrange equation at the fluid-solid boundary for minimizing the total free energy $F[\phi]+\int d s \gamma_{w f}(\phi)$. That is, $L(\phi)=0$ corresponds with the equilibrium (static) condition where $\partial \phi / \partial t+\mathbf{v} \cdot \nabla \phi=0$. Therefore, to the first order, the boundary relaxation dynamics of $\phi$ are plausibly assumed to be proportional to a nonzero $L(\phi)$ :

$$
\frac{\partial \phi}{\partial t}+\mathbf{v} \cdot \nabla \phi=-\Gamma[L(\phi)]
$$

where $\Gamma$ is a phenomenological parameter.

Motivated by the methods presented in $[19,20]$, a second order scheme is designed to solve the $\mathrm{CH}$ hydrodynamic model $(3.2),(3.3),(3.4),(3.5)$ with verified exponential convergence in time to the steady state. Besides those parameters which can be directly obtained from MD simulations, $M$ and $\Gamma$ are treated as fitting parameters, determined by comparison with MD results (values given in the figure legend). In figure 3.1, we show that the continuum model can indeed quantitatively reproduce the interface and velocity profiles from MD simulations, including the near-total slip $\left(v_{x} \simeq 0\right)$ of the CL and the fine features in the molecular scale vicinity of the CL. We wish to emphasize that for the comparison with the symmetric case, the parameters in the continuum model, including those in the GNBC, are directly obtained from the MD simulations, whose velocity profiles are then fitted by those from the hydrodynamic calculations with optimized $M$ and $\Gamma$ values. Thus the comparison with the asymmetric Poiseuille flow, with $\beta_{2}$ directly evaluated from MD simulation data, is without additional adjustable parameters. The above results verify that the boundary conditions and the parameter values are local properties and hence applicable to flows with different macroscopic conditions. The remarkable overall agreement affirms the validity of the GNBC and the hydrodynamic model, as well as justifies the replacement of the diffuse fluid-solid boundary (force density) by a sharp boundary.

\section{Concluding remarks}

In summary, we have found for the first time the boundary condition that yields near-complete slipping of the CL, in good agreement with MD results on the molecular scale. These results open the door to efficient simulations of nano- or microfluidics involving immiscible components, as well as to macroscopic immiscible flow calculations, e.g., in porous media, that are physically meaningful at the molecular level [21]. It should also be noted, however, that the present continuum formulation can not calculate fluctuation effects that are important in MD simulations. Long range interactions, e.g., those due to van der Waals interactions, have also been ignored. The latter is potentially important in the calculations involving wetting layers.

Acknowledgments. Partial support from HKUST's EHIA funding and Hong Kong RGC grants HKUST 6176/99P and 6143/01P is hereby acknowledged. 

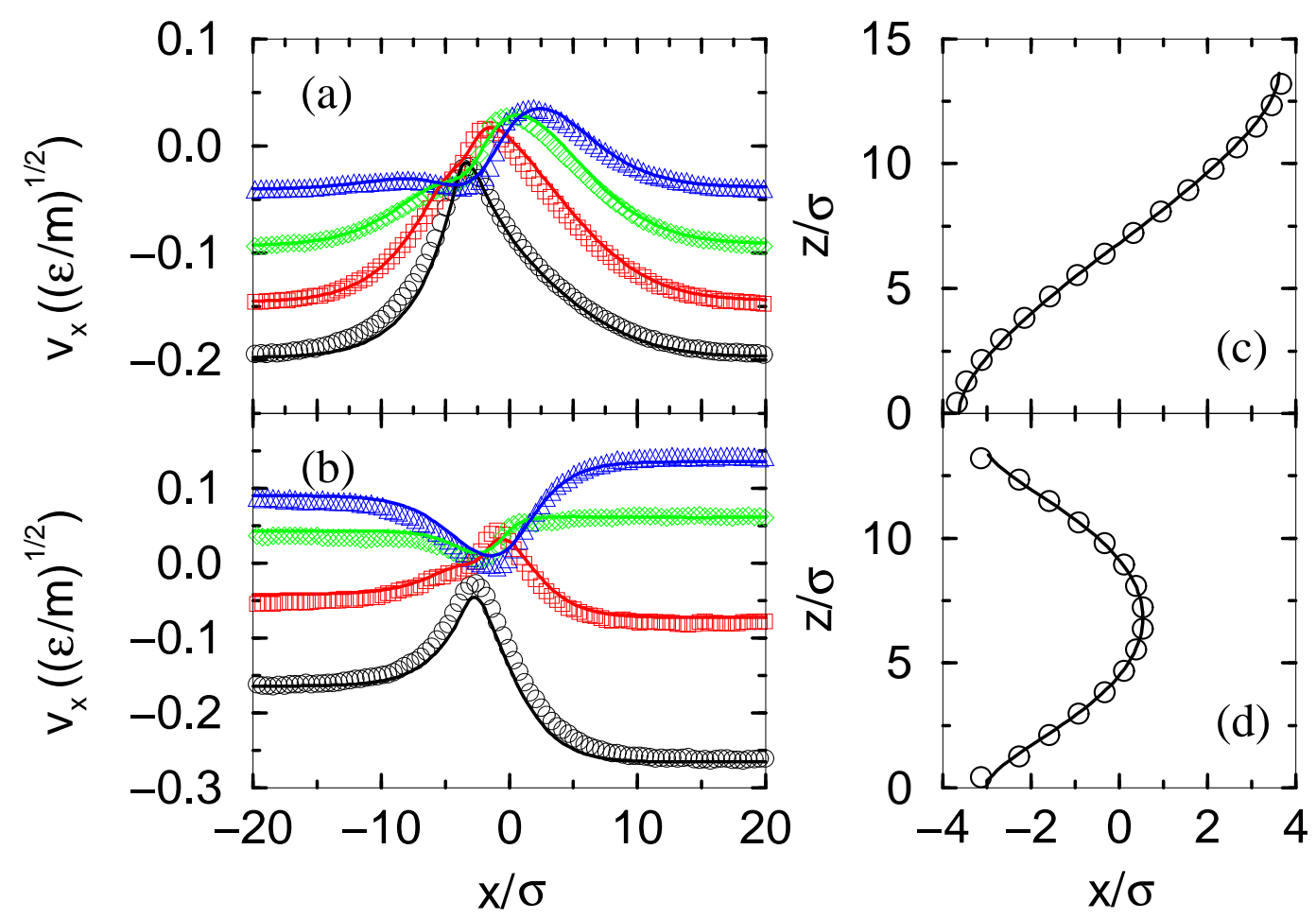

FIG. 3.1. Comparisons between the $M D$ (symbols) and the continuum hydrodynamics (solid lines) results, the latter calculated with the GNBC and values of $M=0.023 \sigma^{4} / \sqrt{m \epsilon}$ and $\Gamma=$ $0.66 \sigma / \sqrt{m \epsilon}$. (a) The $v_{x}$ profiles for the Couette flow in the symmetric case $\left(V=0.25(\epsilon / m)^{1 / 2}\right.$ and $H=13.6 \sigma)$ at different $z$ planes. The profiles are symmetric about the center plane; hence, only the lower half is shown for $z=0.425 \sigma$ (black circles), $2.125 \sigma$ (red squares), $3.825 \sigma$ (green diamonds), and $5.525 \sigma$ (blue triangles). (b) The $v_{x}$ profiles for the Poiseuille flow in the asymmetric case $\left(H=13.6 \sigma\right.$, the two walls moving at a constant speed $V=0.51(\epsilon / m)^{1 / 2}$ in the $-x$ direction in order to maintain a time-independent steady-state interface, with $m g_{\text {ext }}=0.05 \epsilon / \sigma$ applied in the $x$ direction) at different $z$ planes. The profiles are symmetric about the center plane; hence, only the lower half is shown for $z=0.425 \sigma$ (black circles), $2.125 \sigma$ (red squares), $3.825 \sigma$ (green diamonds), and $5.525 \sigma$ (blue triangles). For the boundary layers, $v_{x}=0$ means complete slip. (c) and (d) The interface profiles corresponding to $(a)$ and $(b)$, respectively. The symbols represent the timeaveraged interface profiles from $M D$, defined by $\rho_{1}=\rho_{2}(\phi=0)$. The black solid lines are the interface profiles calculated from the continuum hydrodynamic model with the GNBC.

\section{REFERENCES}

[1] C. Huh and S.G. Mason, The steady movement of a liquid meniscus in a capillary tube. J. Fluid Mech., 81:401-419, 1977.

[2] E.B. Dussan, V., On the spreading of liquids on solid surfaces: static and dynamic contact lines. Ann. Rev. Fluid Mech., 11:371-400, 1979.

[3] J.F. Joanny and P.G.de Gennes, A model for contact angle hysteresis. J. Chem. Phys., 81:552$562,1984$.

[4] P.G.de Gennes, Wetting: statics and dynamics. Rev. Mod. Phys., 57:827-863, 1985.

[5] R.G. Cox, The dynamics of the spreading of liquids on a solid surface. Part 1. Viscous flow. J. Fluid. Mech., 168:169-194, 1986.

[6] J. Koplik, J.R. Banavar, and J.F. Willemsen, Molecular dynamics of poiseuille flow and moving contact lines. Phys. Rev. Lett., 60:1282-1285, 1988. 
[7] J. Koplik, J.R. Banavar, and J.F. Willemsen, Molecular dynamics of fluid flow at solid surfaces. Phys. Fluids A, 1:781-810, 1989.

[8] P.A. Thompson and M.O. Robbins, Simulations of contact-line motion: slip and the dynamic contact angle. Phys. Rev. Lett., 63:766-769, 1989.

[9] P.A. Thompson, W.B. Brinckerhoff, and M.O. Robbins, Microscopic studies of static and dynamic contact angles. J. Adhesion Sci. Tech., 7:535-554, 1993.

[10] M.Y. Zhou and P. Sheng, Dynamics of immiscible-fluid displacement in a capillary tube. Phys. Rev. Lett., 64:882-885, 1990.

[11] G.J. Merchant and J.B. Keller, Contact angles. Phys. Fluids A, 4:477-485, 1992.

[12] Blake first proposed that the uncompensated Young stress contributes to the relative slipping, in the context of an adsorption/desorption model. See T. D. Blake, Dynamic contact angles and wetting kinetics, in Wettability (ed J.C. Berg) 251-309 (Marcel Dekker, Inc., 1993).

[13] H.Y. Chen, D. Jasnow, and J. Vinals, Interface and contact line motion in a two phase fluid under shear flow. Phys. Rev. Lett., 85:1686-1689, 2000.

[14] D. Jacqmin, Contact-line dynamics of a diffuse fluid interface. J. Fluid. Mech., 402:57-88, 2000.

[15] For technical details of the MD simulations, we followed those described in M. Allen and D. Tildesley, Computer Simulation of Liquids (Clarendon, New York, 1987).

[16] J.H. Irving and J.G. Kirkwood, The statistical mechanical theoryof transport processes. IV. The equations of hydrodynamics. J. Chem. Phys., 18:817-829, 1950.

[17] P.A. Thompson and S.M. Troian, A general boundary condition for liquid flow at solid surfaces. Nature, 389:360-362, 1997.

[18] J.W. Cahn and J.E. Hilliard, Free energy of a nonuniform system. I. Interfacial free energy. J. Chem. Phys., 28:258-267, 1958.

[19] H. Johnston and J.G. Liu, Finite difference schemes for incompressible flow based on local pressure boundary conditions. J. Comput. Phys., 180:120-154, 2002.

[20] Weinan E, Numerical Methods for Viscous Incompressible Flow: Some Recent Advances. Advances in scientific computing, p. 29 (Science Press, 2001).

[21] Nanometer-scale details may be resolved through the grid iteration method without significantly compromising the calculation efficiency. See W. Ren and X.P. Wang, An iterative grid redistribution method for singular problems in multiple dimensions. J. Comput. Phys., 159:246-273, 2000 . 